Bol. Acad. peru. leng. 48. 2009 (125-137)

\title{
DEFINICIONES EN LAS PAPELETAS LEXICOGRÁFICAS DE RICARDO PALMA
}

\author{
Andrés Napurí \\ Pontificia Universidad Católica del Perú
}

Fecha de recepción:

$19 / 09 / 2009$

Fecha de aceptación:

$12 / 11 / 2009$

\section{Introducción}

La lengua ha sido y será siempre objeto de discusión y controversia. Ha sido, justamente, una controversia lo que llevó a Palma a discutir las voces del diccionario de la Real Academia Española. Para Palma, existían cerca de dos mil setecientas voces que no eran reconocidas y por consiguiente había un vacío en la descripción de la lengua; vacío que él buscó dar contenido y fue por eso que terminó editando sus Papeletas Lexicográficas.

Ahora, acá surge el problema que resulta interesante para este texto. ¿Cómo se le da contenido a una palabra? El contenido, en este sentido, no es sólo el significado que le dé uno como hablante, a la palabra. Palma, como persona que busca generar conocimiento científico, trata 
de desarrollar métodos para abordar los distintos problemas que tienen las diversas naturalezas de las palabras. Es decir, no existe una única manera de realizar una definición lexicográfica y Palma era consciente de eso. A esta primera pregunta, es importante señalar otra consideración y es ¿cómo dar contenido a aquellas palabras que no corresponden a la tradición hegemónica?

Las Papeletas lexicográficas son una pieza más de la confrontación que señala Derrida y que también recoge Palma. Palma creía que la acción de la Academia podía vigorizar vínculos más que los mismos gobiernos y de alguna forma conformar una identidad como pueblo hispano-americano (Palma, 2003: X). Sin embargo, tras el rechazo de las entradas léxicas; la lengua compartida se convierte y es presentada como la lengua del otro. Siendo así el trabajo en estas papeletas no sólo un trabajo lexicográfico; sino también un punto de vista sobre cómo se percibe España en América. Así deriva la crítica de Palma hacia la Academia y esa es la naturaleza de la segunda pregunta planteada y que resulta necesario responder. Para partir de la respuesta y así explicar parte del método de Palma en la descripción del léxico.

Teniendo esto claro, se puede continuar ahora con el análisis.

\section{Lengua}

La lengua ha sido y será siempre objeto de discusión y controversia. Para comprender la postura que asume Palma al confrontarse a la Academia, es importante reconocer primero en qué contexto histórico se encontraba y a partir de ese reconocimiento dar una reflexión.

Palma consideraba que las políticas españolas eran erradas; España no fortalecía sus nexos con América en general. Francia e Inglaterra sacaban un mayor provecho de Perú con el comercio. De manera que las nuevas generaciones tenían a estos países como referencia, mientras que eran indiferentes con España (Huisa 2003: 100). Palma no podía ser indiferente con España, pues consideraba que «la lengua de Castilla» era 
el símbolo de una relación estrecha que se debía salvar. De ahí que Palma señale: «[...] Generalizada creencia es, en América, la de que España no nos perdona el que hayamos puesto casa aparte, desprendiéndonos de su maternal regazo [...]»; es una forma de señalar que la visión negativa hacia España parte de un resentimiento y que España puede ser un modelo.

Si bien por un lado Palma busca fortalecer el nexo entre España y América para el bienestar de ambas partes; como ya se dijo, España mantenía políticas erradas con América. Palma criticaba, por ejemplo, que no se reconocieran los americanismos hablados por millones y sí provincialismos hablados por pocos miles; por otro lado, también consideraba que se "hería susceptibilidades de nacionalismo» al señalar <Méjico> y no <México> (Huisa 2003: 101). Este tipo de políticas del lenguaje resultaban ser enajenantes y en ese sentido Palma comienza un trabajo por la oficialización de las voces americanas.

Así pues Palma aborda «[...] el tema del léxico hispanoamericano en la relación entre España y Perú, desde un punto de vista social antes que lingüístico [...]» (Huisa 2003: 99). Sin embargo, si bien la motivación no es precisamente lingüística y su trabajo parte con un sesgo particular de reconocimiento, su discurso no deja de ser crítico. Palma desarrolla criterios de recolección de datos, critica Academia por entradas que no poseen uso generalizado y critica el purismo y actitud conservadora. Considera, además, que las nuevas voces son necesarias en tanto responden a necesidades cognitivas de los hablantes y que el purismo no va a la par con el progreso y conocimiento (Huisa 2003: 107).

Esta descripción de la situación de la lengua para Palma se puede resumir de la siguiente manera, siguiendo a Huisa: «[...] La postura de Palma se caracteriza por la permanente contradicción entre la aceptación de la autoridad de la Academia y del Diccionario, y la negativa de ella de aceptar los vocablos que se utilizan con frecuencia en América [...]» (Huisa 2003: 102). Palma, entonces, encuentra sujeta su labor lexicográfica a una tradición discursiva que es distinta a la propia a pesar de compartir el mismo idioma. 
La pregunta ahora es, ¿es necesaria reconstruir una tradición para tener una base sobre la cual dar sentido al significado de las palabras? Indudablemente sí, o al menos se ha dado así históricamente. El Círculo de Praga es un ejemplo al reconstruir la historia de las lenguas eslavas para así poder hablar de una lengua eslava literaria. El punto es: reconstruir una tradición sirve, entre otras cosas, para asignar valores a una lengua. En la caso del trabajo lexicográfico es útil para dar un vocabulario intelectualizado.

Es justamente la reconstrucción de la tradición lo que ha permitido el uso de definiciones por autoridad. Estas son definiciones que se explican por el uso que le dan los autores reconocidos a las palabras en cuestión. La Academia, al no reconocer las entradas léxicas de Palma, las sitúa como variedad no estándar. Lara reflexiona sobre qué se entiende por lenguas no estándares y señala que «[...] es lengua no-estándar la que no dispone de un rico vocabulario intelectualizado y, en esa medida, no es capaz de prestarse (sino limitadamente) a la transmisión del conocimiento y la civilización [...]» (Lara 2004b: 119). Esta descripción se puede dar análogamente a las variedades no estándares, como es el caso de Palma. No está de más señalar que la lengua estándar es una construcción histórica y artificial, así como todo aquello que no sea estándar lo es.

Entonces, la pregunta sobre cómo dar contenido a aquellas palabras que no corresponden a la tradición hegemónica comienza a responderse con la construcción de una tradición. Esta tradición permite intelectualizar los vocablos (por decirlo de alguna forma) y así colaborar a que sean parte del conocimiento cultural de una lengua. En la creación de una tradición y en la inclusión de nuevo léxico se ve la lengua favorecida.

Un aspecto importante en este criterio de tradición como forma de reconocimiento, es que en el caso de las Papeletas lexicográficas no corresponden al uso oral. Esto es muy interesante al ser Palma reconocido por las Tradiciones Peruanas, obra que recoge las costumbres y lo cotidiano. Lara realiza una observación muy interesante y es que 
las prácticas orales de las comunidades lingüísticas y todas esas formas líricas del relato popular son esperables de una lengua no-estándar (Lara 2004b: 122). Es por eso que Palma no utiliza los registros orales para dar autoridad sobre el uso de un vocablo. Más bien, coincide históricamente con otros trabajos lexicográficos y parte de textos escritos de autores para dar precisión a las palabras. Eso no quiere decir que deje de lado el uso que le da los hablantes a las palabras; sí lo toma en cuenta, pero utiliza este criterio más que nada para señalar la extensión de un registro entre los hablantes.

Por otra parte, tener a los textos escritos le permite hacer una distinción mejor entre variedades y referirse así no sólo a una tradición americana que no es reconocida por la Academia en España; sino a una serie de tradiciones de variedades nacionales. Lo que termina dando mucho más peso a la situación que busca resolver Palma. Porque a fin de cuentas, lo que llega a elaborar es una conceptualización de varios discursos relevantes que conforman una comunidad de millones.

Para resumir esta parte, Palma reconoce que es importante crear una tradición que sea base de los vocablos que registra. Además, debe ser una tradición escrita, por su fácil identificación con la tradición hegemónica. Por otro lado, al ser una tradición escrita es más fácil de asociar y de generalizar. De esta manera, el discurso de Palma no recoge una única tradición, sino una serie de tradiciones nacionales; dándole peso a sus palabras frente a la postura de la Academia. Esta tradición es necesaria para poder crear un espacio de reflexión acerca de las entradas léxicas no registradas y así darles contenido que pueda ser intelectualmente relevante.

De esta manera se concluye la ubicación general de la lengua que Palma busca describir dentro de su espacio social e histórico. A esta altura del texto, los intereses y la percepción de las entradas léxicas por parte de la Academia así como por parte de Palma deben estar claros. Una vez esto claro, es posible analizar los criterios utilizados por Palma al abordar las entradas léxicas y así entender cómo le da contenido. 
Dicha descripción se caracteriza por tener distintas estrategias; para así poder dar una interpretación que ayude a la comprensión. La complicación agregada de estas palabras, al no ser parte del estándar, es que aparte de que se busca recuperar todos los matices en su descripción, la descripción de hace utilizando el estándar. La otra lengua.

\section{Estrategia:}

Como se anunció en el capítulo anterior, en este espacio se desarrolla los distintos criterios manejados por Palma al momento de realizar la descripción de las entradas léxicas en su libro Papeletas lexicográficas.

Palma buscaba escribir definiciones. Dentro de estas definiciones, se pueden reconocer hasta dos momentos. Un primer momento en que Palma distingue a la palabra descrita y su uso, frente al uso que le atribuye la Academia. Este primer momento tiene una fuerte carga ideológica y se busca corregir a la autoridad. En todo caso, se busca señalar que la palabra que registra el Diccionario, no es la que se usa en América. Esto se puede reconocer, por ejemplo, en la palabra <boleto>:

Boleto - Lo que el Diccionario llama boleta. Nadie, en América, compra boleta de teatro, de plaza de toros ó de ferrocarril, sino boleto. También damos este nombre á una excepción firmada por la autoridad.

Dentro de este primer momento de la descripción, aparte de corregirse a la Academia, también se señala que la voz no registrada corresponde a una tradición nacional. Es tanto así, que incluso aparece una voz en primera persona plural, como en el caso de <cachetada>: «[...] No decimos, como en España, dar un cachete, sino dar una cachetada [...]» (Palma 2003). Si no sucede esto, se hace referencia al uso de la palabra en algún país de América y se le asigna una tradición, puede ser una crónica. En última instancia se hace énfasis en el autor, en algunos casos son autores españoles, en otros casos son autores americanos. Lo importante en este punto es la autoridad, dado que «[...] su simbolismo se dirigía a la legitimación de las lenguas literarias [...]»(Lara 1997a: 46). 
En este primer momento, en que se describe el origen de la palabra, también hay espacio para otras voces, no sólo hispanas. Por ejemplo, también ingresa la voz <boycotear> y se justifica su ingreso por no existir en ese momento un «[...] verbo que exprese con más concisión la idea [...]» (Palma 2003). Este comentario de Palma no debe pasar desapercibido. Su interés, está marcado por el reconocimiento de las voces y la construcción de tradiciones nacionales; no obstante, no deja de lado la importancia de las palabras mismas por su uso. Palma hace bastante énfasis en agregar léxico por su estrecha relación con el desarrollo de las sociedades, son puente a nuevos conceptos.

El segundo momento de la descripción lexicográfica que realiza Ricardo Palma es quizá la menos cargada ideológicamente y la mejor desarrollada lingüísticamente. Para la elaboración de este texto se hizo un repaso general de los matices que puede tener la definición lexicográfica.

Palma entendía que para dar el significado de una palabra, no bastaba con dar con un sinónimo del español general. Debía poder describir el cambo semántico de la palabra. Es decir, dar un contexto que ayude a entender qué significa esta palabra realmente. Ante este reto hay que agregar, que Palma no era un lexicógrafo. No poseía las herramientas teóricas para dar un análisis preciso. Cuando uno revisa el libro se da cuenta que en realidad eso no parece haber sido una limitación.

Palma era un intelectual que ya realizaba cierto tipo de trabajo de campo. Él ya debía contar con cierto método y con una intuición ya desarrollada por su mismo acercamiento hacia la lengua. Por ejemplo, para escribir las Tradiciones peruanas, debía tener una noción muy clara sobre la lengua en uso. Poder captar eso, ya supone una estrategia y acercamiento determinado. Acercamiento que bien él podía utilizar al momento de escribir las definiciones. No por gusto, sus definiciones tienen referencias a la lengua en uso o en contextos cómicos como es el caso de <fusilable>. 
Teniendo esto claro, es entendible que el trabajo de Palma frente a las palabras definidas sea tan parejo y en cierta forma metodológico.

De hecho, resulta interesante ver cómo Palma enfrenta convenciones. No da por sentado que el sentido de una palabra sea lo estipulado, sino que busca entender el significado de lo que se dice. Por ejemplo en el caso de la palabra <discante>, Palma critica a la Academia por tomar sólo una acepción; agregando él que también se usa para señalar una acción sin objetivo. No sólo a nivel de acepciones, también en un nivel general como en <desvestirse>. Esta palabra la distingue de <desnudarse> e indica que no son sustantivos y que la primera es cambiarse de vestido, mientras que la segunda es quedarse en «[...] traje paradisiaco $[\ldots] »$.

Al no dar las convenciones por sentado Palma rescata al hablante. $\mathrm{Al}$ final es el uso el significado de la palabra. Como decía Wittengstein: «lo que entendemos los hablantes como el conjunto de proposiciones con que se explican y describen las características o cualidades de algo, sus rasgos, sus límites» (Lara 2004a: 38).

Partiendo del uso, no sería consecuente manejar definiciones nomenclaturistas. Estas definiciones se caracterizan por tratar a la palabra como un «[...] soporte material de la referencia a un objeto [mental] o a la experiencia de la vida [...]» (Lara 2004a: 41). En ese sentido, las palabras son sólo nombres de cosas o ideas. Si el criterio de uso era importante para dar sentido a las palabras, Palma parte de una concepción consustancialista. Es decir, toma la palabra como signo y es el uso lo que define los significados que pueda tener (Lara 2004a: 43). Coincide entonces con que cada palabra es una construcción.

Un ejemplo de este interés por la «sustancia» es <cocacho>, que se define como: «[...] Golpe que con el puño se da en la cabeza - Frejol cocacho, el fríjol que conserva alguna dureza por mal cocido [...]». Como se puede notar, el primer sentido parece que se ha extendido al segundo. El golpe sobre el hueso duro del cráneo se ha extendido a la dureza del frejol por estar mal cocido. Esa extensión del significado lo hacen los hablantes; es parte de su ingenio, al jugar con el campo de uso que pueda tener un 
signo. Palma hace bien al recoger este uso ingenioso y reconocerlo como parte de uso de la lengua.

Palma posee numerosas entradas con esa característica; no obstante, también posee entradas léxicas más apegadas al signo mismo. Aunque son raras las palabras en las que deja de lado el criterio de uso o de tradición. Por ejemplo, la palabra <pretensioso>, es un buen caso de definición de signo. La descripción de la palabra llega a sustituirla (así se entiende a una definición de signo): "[...] Persona que alardea de gran competencia y méritos ó que aspira á aventajar á otros en saber, belleza, acciones, etc. [...]». Sin embargo, como la carga de la tradición es muy importante, la definición continúa. Palma agrega que el registro de esta palabra es antiguo y que en España se encuentra registrada como <pretencioso>, lo cual no tiene real razón de ser por el origen de la palabra.

Otra característica muy interesante en el desarrollo de las definiciones de Palma, es que son de lectura sencilla. Uno podría esperar que siendo él un académico y que prepare un texto para los académicos de la península, el contenido podría ser difícil de procesar. Esto no se da, el uso de autoridades son más que nada para ejemplificar el uso; no busca ser críptico ni carga las Papeletas lexicográficas con información filológica innecesaria. Sólo la señala en caso sea relevante para la discusión, como en <pretensioso>. Fuera de eso, busca dar definiciones de la sustancia referida por la palabra. No tiene reparo en describir lo que se busca explicar, como en <acholarse>. Para esta palabra, por ejemplo, no sólo brinda sinónimos, sino que explica el su origen de manera breve y concisa: «[...] Este americanismo se funda en la timidez característica de nuestros indios, cuando se ven forzados a hablar con la autoridad $[\ldots] »$.

Con seguridad, parte de esa facilidad que tiene para generar definiciones que sean de fácil lectura es por su trabajo como escritor y como recolector de tradiciones. Palma manejaba estilos diferentes dependiendo de qué quiera reflejar. Además, como recolector de tradiciones, debía tener una sensibilidad particular y poder percibir y manejar distintos registros de uso de la lengua. 
Otras definiciones manejadas por Palma son las definiciones ostensivas, que buscan explicar la palabra fuera de ella. Llaman la atención del lector hacia el mundo y muestran que lo referido por la palabra no se encuentra en su estructura. Ese es el caso de palabras como <anticucho> o la planta <achira>. Son realidades tan naturales o sociales que sus descripciones deben ser lo más prácticas posible y referirse al mundo.

Otro tipo de definiciones que aparecen bastante en las Papeletas lexicográficas, son las definiciones por ecuación sémica. Lara señalar que hay verbos que se utilizan para dar un sentido a los tipos de definición; verbos que van de la mano con las maneras en que la lexicografía practica la definición (Lara 2004a: 52). En el caso de Palma, estos verbos no aparecen de manera explícita, pero sí hay palabras que utilizar él para generar un tipo de definición.

Una de estas palabras es «persona». El uso de la palabra "persona», va de la mano con nombres con un fuerte grado de agencia, como <exacerbador>, <exhibidor>, <jubilador>. Son personas que pueden hacer algo o afectan en algo al resto. Este uso de "persona» no es estricto. También se utiliza en personas que realizan actividades, pero que no necesariamente afectan a los demás, como <excursionista> o <realizador>.

Otra de las palabras que utiliza bastante Palma como introducción a sus definiciones, es la palabra «que». Es, seguramente, la palabra que más utiliza para comenzar una entrada léxica. No suele utilizar para referirse a individuos o características de algo, como 〈retificable>: «[...] que puede ratificarse [...]». Estas definiciones por lo general son bien breves y son muy numerosas. $\mathrm{Al}$ comienzo pueden resultar extrañas, por no tener el contenido de otras definiciones ya mencionadas. No por eso dejan de ser interesantes.

Lara (2004b) señala que al momento de trabajar con variedades no estándares se busca llenar un vacío y se busca recoger la mayor cantidad de registros posibles. Con la intención de darle peso a la recolección hecha. En parte, esa es la naturaleza de los diccionarios de americanismos, en los 
que se busca contrastar las voces registradas frente al resto (Zimmermann 2003). No obstante, esta no parece ser la razón del registro de estas voces. En el caso de Palma, en la mayoría de las palabras no se les asigna ninguna tradición particular, ni ningún origen particular. Por otra parte, Palma buscaba que estas voces sean incluidas en el Diccionario. Las edita por separado al no poder ingresarlas, pero ese ya es un conflicto ideológico de otro tipo.

No parece ser coincidencia que siempre el «que» refiera a un acto o peculiaridad. Salvando distancias, parece semejarse a un tipo de definición aristotélica (Lara 1997b: 206), quizá un tanto simple; pero se refiere a la esencia de la palabra y su real contenido. La palabra que es definida seguida por un «que», refleja en realidad que hay más léxico de dónde vino esta palabra y la derivación podría producir otras nuevas palabras. Acá hay que tener en consideración que es justamente un libro que presenta nuevas palabras para el Diccionario dado su uso extendido. Si esta lectura es válida, Palma no sólo estaría prestando atención a la tradición y al hablante con la lengua en uso. También estaría reflexionando sobre las posibilidades del lenguaje para generar nuevas entradas. Como bien señaló Huisa, el ingreso de nuevo léxico siempre preocupó a Palma, ya que para él el léxico conforma la manera en que se construye y desarrolla una sociedad.

Un límite de esta lectura sería que en muchos casos, el «que» introductorio se refiere a un individuo o una persona que realiza una labor. Se puede afirmar que era una manera de referirse a oficios o calificativos de uso general. Lo curioso, es que la labor que se realizaba o el origen del calificativo también figura en las Papeletas lexicográficas. Puede resultar mucho más cómodo, referirse sólo a lo segundo y dejar de lado la derivación morfológica. Podría ser cómodo, pero esa no es la realidad del libro, el libro muestra adrede esta derivación, muestra la producción del lenguaje y las posibilidades de uso.

Hasta ahora se han ido señalando algunas herramientas que manejó Palma para escribir las Papeletas lexicográficas. Con las ya mencionadas se puede dar una lectura general al libro y notar que efectivamente él 
maneja todas estas estrategias. Lo cual es una muestra de su habilidad e intuición para manejar criterios lexicográficos que probablemente no conocía. La experiencia en el trabajo de campo de las Tradiciones peruanas le debió servir abordar sistemáticamente las palabras del léxico que iba registrando. Además, de saber marcar dos momentos en su trabajo; el (1) cargado ideológicamente sobre el problema de la lengua y el (2) trabajo lingüístico y filológico.

Para ir concluyendo, la imagen de la definición de Palma es bastante rica. Por un lado, por saber abordar un problema de hegemonía y buscar dar crédito a léxico usado en América. Brindar una tradición literaria y utilizar nuevas autoridades. Por otro lado, por darle importancia al léxico como conocimiento y cultura. Además, por desarrollar una estrategia sólida al momento de trabajar las entradas léxicas. Estrategia que no sólo resalta en aspectos formales, sino también por su claridad y sus fuertes coincidencias con criterios lexicográficos que se manejan al día de hoy. Logrando así resolver las dos inquietudes principales de este texto; al enfrentar a una tradición que se presenta como otra y al dar contenido a palabras.

\section{BIBLIOGRAFÍA}

DERRIDA, Jacques. El monolingüismo del otro. Traducción de Horacio Pons. Buenos Aires, Manantial, 2002.

HUISA, José Carlos. Introducción al estudio de la lexicografía hispanoamericana y análisis de las obras lexicográficas del español del Perú a partir de sus prólogos. Tesis de licenciatura en Letras y Ciencias Humanas con mención en Lingüística Hispánica. Lima, PUCP, Facultad de Letras y Ciencias Humanas, 2003.

LARA, Luis Fernando. "El papel del léxico". En Teoría del diccionario monolingüe. México D.F., El Colegio de México, 1997a. 
- "La definición lexicográfica como construcción cultural". En Teoría del diccionario monolingüe. México D.F.,El Colegio de México, 1997b.

"La definición lexicográfica como reconstrucción del significado”. En Teoría del diccionario monolingüe. México D.F., El Colegio de México, 1997c.

. "El sentido de la definición lexicográfica". En De la definición lexicográfica. México D.F., El Colegio de México, 2004a.

. "La descripción del significado del vocabulario noestándar”. En De la definición lexicográfica. México D.F., El Colegio de México, 2004b.

PALMA, Ricardo. Papeletas Lexicográficas. Lima, Academia Peruana de la Lengua, USMP, 2003.

ZIMMERMANN, Klaus. "El fin de los diccionarios de americanismos. La situación de la lexicografía del español de América después de la publicación de los Diccionarios contrastivos del español de América". Revista internacional de lingüistica iberoamericana. Madrid, Número 1, pp. 71-83, 2003.

\section{Correspondencia:}

\section{Andrés Napurí}

Estudiante de Lingüística Hispánica en la Pontificia Universidad Católica del Perú.

Correo electrónico: a.napuri@pucp.edu.pe 